\title{
Relational algebraic approaches to decision making under uncertainty
}

\author{
$\underline{\text { Anushka Chandrababu }}{ }^{\text {a }}$, Abhilasha Aswal a and G N Srinivasa Prasanna ${ }^{\text {a }}$ \\ ${ }^{a}$ International Institute of Information Technology - Bangalore, India \\ Email:anushka.babu@iiitb.org
}

\begin{abstract}
Uncertainty is so ubiquitous in most real world problems that understanding the fundamental nature of uncertainty is important. Stochasticity has been shown to be only one of the many dimensions of uncertainty. Uncertainty can be of many types and different methodologies for decision making are applicable in different cases. In cases where probability distributions of uncertain parameters cannot be ascertained with great confidence, robustness of strategies is more important than optimality and hence the robust optimization paradigm is more appropriate.
\end{abstract}

We present a simple polyhedral representation of uncertainty based on robust optimization that can be generated through specification of constraints or easily derivable from historical data with underlying uncertainty. Such representations are a convenient way to summarize large datasets and lead to tractable ways of comparing alternative scenarios or assumptions. We also present a set theoretic relational algebra to qualitatively compare these polyhedral models (polytopes) or alternative sets of uncertainty. The relational algebra arises because of the non-zero measure of these convex polyhedral objects - these are aggregates. We define the subset, intersection and disjoint relational algebraic operators to compare alternative scenarios or assumptions. An intersecting relationship between two polyhedral models based on assumptions means that there is commonality between the two assumptions. Similarly, if one is a subset of the other, then its underlying data is more specific than the other.

To facilitate analysis of a large number of such different scenarios with underlying uncertainty sets, we have also developed a novel database with facilities to store and perform such set theoretic analysis of alternative future scenario sets efficiently and easily through simple queries. We call our database as $\boldsymbol{C M d B}$ or Convex Model Database. The database can be coupled to any decision support system to help in decision making and analysis under uncertainty. Hence, with our approach, it is easy to - quantitatively and qualitatively compare one set of future scenarios against another, resolve conflicting future estimations, optimize over such alternatives and relate the optimization outputs to the inputs. We can do this in a tractable manner with a wide variety of constraints and assumptions about the future. We show this in the paper with our examples from supply chain and transportation systems.

Keywords: $\quad$ Decision making under uncertainty, Convex modelling, convex model database, decision support systems 


\section{INTRODUCTION}

Uncertainty is an integral part of many optimization and planning problems and one cannot ignore the effects of uncertainty, as such a disregard might lead to highly sub-optimal or even infeasible solutions. Even small perturbations in the nominal values of data can result in highly infeasible solutions (Ben-Tal and Nemirovski 2000). The classical paradigm of stochastic programming is to model uncertainty as a probability density. However, in many practical situations, it is exceedingly difficult to accurately describe probability distributions of uncertain parameters. The robust optimization paradigm can be used in such cases. Robust methodology models uncertainty as ranges or convex sets, while one tries to protect against infeasibilities and nothing more is assumed about the uncertainty sets. An "uncertainty immunized" or "robust feasible" solution is one which remains feasible no matter what the realization of the data is. "Robust optimal" solution is the best solution among all the uncertainty immunized solutions.

Traditionally, uncertainty was understood to have one dimension, nature of uncertainty. This can be - either uncertainty due to variability inherent to a system under consideration (also known as aleatoric uncertainty), where probabilities are known; or uncertainty due to limited data and/or knowledge (also known as epistemic uncertainty), where the probabilities are unknown. At the present time, uncertainty is understood to have many more dimensions (Morgan 2009), (Bradley 2014), (Walker 2003), (Spiegelhalter 2011; Riesch 2012), such as the location of uncertainty within the system model, different levels of uncertainty between the extremes of complete certainty and total ignorance, etc. Higher level uncertainties have alternatively been termed as deep uncertainty (Lempert 2006), or severe uncertainty (Ben-Haim 2001).

\subsection{Decision making under uncertainty}

When the uncertainties are well defined, the focus is on identifying optimal strategies and methods such as those based on Bayesian decision analysis are applied. However, under deep uncertainties, the robustness of strategies is more important than optimality and traditional decision making methods looking for optimality are of limited use. In such situations, it is imperative that decisions be made while considering a wide range of alternative future scenarios and choose strategies that are robust. Scenario planning (Van der Heijden 1996), is the classical technique to find robust policies. Bankes (Bankes 1993), described a methodology called Exploratory modeling and analysis (EMA) and Lempert et. al. (Lempert et. al. 2006) proposed an approach called Robust decision making (RDM) for decision making under deep uncertainty that is based on EMA. BenHaim (Ben-Haim 2001) proposes a decision making framework under deep uncertainty based on informationgap models of uncertainty. The study of alternative future scenarios is equivalent to an analysis of alternative assumptions or beliefs held by a decision maker. It would help a decision maker to compare one set of beliefs or assumptions against another to resolve potentially conflicting predictions, about the future. Our work focuses on such an analysis while quantitatively analyzing assumptions and beliefs. In section 1.2 we briefly describe our representation of uncertainty and a novel database that supports decision making within our framework, along with the specific contributions of our work.

\subsection{Our representation of uncertainty and the Convex Model Database}

Our uncertainty sets are modeled as convex polyhedra consisting of intuitive and simple linear constraints that are derived from historical time series data (sums and differences of demands, capacities, supplies, inventories etc). In economic and OR applications these constraints have economic meaning and reflect substitutive/complementary behavior. As an example, for demand parameters in a supply chain, the substitutive, complementary and aggregate behavior can be expressed through the following constraints:

- Substitutive: $L B_{1} \leq d_{1}+d_{2} \leq U B_{1}$ - if one demand increases, the other must decrease and vice versa.

- Aggregates: $L B_{2} \leq \sum\left\{d_{i}\right\} \leq U B_{2}$ - the total demand is bounded.

- Complementary: $L B_{3} \leq d_{1}-d_{2} \leq U B_{3}$ - if one demand increases, the other increases with it and vice versa.

The advantage with using this polyhedral representation of uncertainty is that it is intuitive to the decision maker (especially in economic and OR applications). This representation allows us to develop high speed methods to analyze alternative sets of constraints using a novel set theoretic relational algebra described in Section 2. To facilitate analysis of a large number of such uncertainty sets, we have also developed a novel database with facilities to store convex models and perform such set theoretic analysis of alternative scenario sets efficiently and easily through simple queries. We call this database a Convex Model Database (CMdB) which can be queried by Decision Support Systems.

Our specific contributions include: 
- A simple polyhedral representation of uncertainty based on robust optimization that can be generated through specification of constraints or easily derivable from historical data in order to do our analysis in a tractable way.

- A set theoretic relational algebra to qualitatively compare alternative sets of uncertainty.

- A database with facilities to store and query convex uncertainty sets and that can be coupled to any decision support system to help in decision making. We show the capabilities of the database with examples from applications.

The rest of the paper is organized as follows. Section 2 describes our novel database for comparing alternative assumptions along with the set theoretic relational algebraic operations that can be performed on convex uncertainty sets. Section 3 shows examples of decision making in applications such as supply chains and transportation systems where the underlying data is uncertain.

\section{CONVEX MODEL DATABASES FOR COMPARISON OF ALTERNATIVE ASSUMPTIONS}

The $\mathrm{CMdB}$ is a database to store and manipulate convex models efficiently for a Decision Support System. In a CMdB, a table or relation $R=\left(A_{1}, A_{2} \ldots A_{n}\right)$, where $A_{i}$ are the attributes and a tuple $t$ in relation $R$ will have $t\left[A_{i}\right]=C$ where $C$ is a convex model such that for any two points $x_{1}, x_{2} \in C, 0 \leq \theta \leq 1, \theta x_{1}+$ $\left(1-\theta x_{2}\right) \in C$. Though the database in general can be used for all convex models, our initial work is limited to polyhedral models or polytopes. We consider the half plane representation of polytopes where, $\varepsilon$ is an affine Euclidean space of finite dimension $n$, and polytope $P=\bigcap_{i=1}^{p} H_{i}$ of $\varepsilon$ is defined as the intersection of a finite number of $p \geq 1$, of closed half spaces, $H_{i}$. In the case of polytopes, an attribute $A_{i}$ could be the volume of the polytope, the amount of information in terms of bits contained in the polytope computed using Shannon's entropy (Shannon 1948), etc.

A tuple $t$ in $R$ with $t\left[A_{i}\right]=C$ has $C=\left\{x_{1}, x_{2} \ldots x_{n}, y_{1}, y_{2} \ldots y_{m}\right\}$ where $x_{1}, x_{2} \ldots \ldots x_{n}$ are the sample points used to derive the model and $y_{1}, y_{2} \ldots y_{m}$ are the new unseen data points that lie within the bounded region of the convex polytope. The model thus represents future uncertainty, where the model is the sole representative of all future possibilities. Similarly, models with different assumptions or models with different future possibilities can be stored and compared in the database. To compare different assumptions, we have defined set theoretic relational algebra for convex uncertainty sets. A database is useful when the number of such future possibilities or scenarios is large. The CMdB proves useful in such situations as shown in our example in section 3.4 where analysis on many scenarios can be done by simple querying.

\subsection{Set theoretic relational algebra for convex uncertainty sets}

The relational algebra of convex representations of uncertainty qualitatively compares alternative sets of uncertainty based on high speed computational advances in convex optimization. Convex representations are also a convenient way to summarize large datasets and lead to tractable ways of comparing alternative summaries. The relational algebra arises because of the non-zero measure of the convex objects - these are aggregates. Point data are either equal or not-equal, aggregate data can be disjoint, partially or fully overlapping. This results in a rich relational algebra. Opposed to points, in the case of sets we can have relations such as partial overlap amongst two sets in addition to just equality and inequality. Let us suppose that there are two convex uncertainty sets or data summaries $A$ and $B$. The possibilities for set relations between set $A$ and set $B$ are outlined below:

- Set $A$ is more specific than $B$ and does not include anything that is missing from $B$. In set theoretic language, $A \subset B$.

- $\quad$ Sets $A$ and $B$ are totally different and have no (N-dimensional) parameter values in common. In set theoretic language, $A \cap B=\phi$

- Sets $A$ and $B$ have some assumptions in common, but neither is a subset of the other: $A \cap B \neq \phi$

For polyhedral uncertainty sets, the set relations can be established through direct solution of LPs containing the joint constraint sets. For example, for subsets we can find points in the subset common to both, and points in the superset, and a hyperplane cutting the superset, but not in the subset. For other forms of uncertainty such as ellipsoidal uncertainty sets and probabilistic uncertainty sets, it is much harder to establish the set relations.

\subsection{Comparison with ellipsoidal uncertainty}

For ellipsoids, a direct method of detecting subsetness involves solving a linear matrix inequality formulated using the S-procedure (Boyd and Vandenberghe, 2004). Ellipsoids can be represented as sublevel sets of a quadratic function as follows: 


$$
\begin{gathered}
\varepsilon_{1}=x^{T} F_{1} x+2 g_{1}^{T} x+h_{1} \leq 0 \\
\varepsilon_{2}=x^{T} F_{2} x+2 g_{2}^{T} x+h_{2} \leq 0
\end{gathered}
$$

Where $F_{i} \in S_{++}$and $h_{i}-g_{i}^{T} F_{i}^{-1} g_{i}<0 ; h_{i} \in R, g_{i} \in R^{n}$.

The condition that $\varepsilon_{1}$ is a subset of $\varepsilon_{2}$ can be stated as the following: $\varepsilon_{1} \subseteq \varepsilon_{2}$ if and only if there is a $\lambda>0$ such that:

$$
\left[\begin{array}{ll}
F_{2} & g_{2} \\
g_{2}^{T} & h_{2}
\end{array}\right] \leq \lambda\left[\begin{array}{ll}
F_{1} & g_{1} \\
g_{1}^{T} & h_{1}
\end{array}\right] \Rightarrow\left[\begin{array}{ll}
F_{2}-\lambda F_{1} & g_{2}-\lambda g_{1} \\
g_{2}^{T}-\lambda g_{1}^{T} & h_{2}-\lambda h_{1}
\end{array}\right]
$$

which involves solving a convex optimization. Clearly, relational operators with ellipsoidal uncertainty is computationally heavy, compared to polyhedral uncertainty (which uses only LP's).

\subsubsection{Comparison with stochastic uncertainty}

Stochastic uncertainty introduces non-uniform probability distributions. Relational algebra with non-uniform probability distributions is not easy in general and involves solving integrals. However, we can use feature extraction using (say) neural networks, and do the relational algebra in the feature space, where it is linear, if the features are so designed. One technique to quantify the agreement between two probability distributions is to find the area of the region where the two density functions overlap. Let $p_{1}(x)$ and $p_{2}(x)$ characterize two different specifications of uncertainty with finite support. The area of the overlapping region of the two functions is given by the following integral, also known as the Weitzman's Measure:

$$
\Delta=\int \min \left\{p_{1}(x), p_{2}(x)\right\} d x
$$

Probability distributions with infinite support will generally intersect, but this is not always the case. For example, consider p.d.f's that have nonzero values in $\mathrm{y}=\mathrm{x}$; and $\mathrm{y}=\mathrm{x}+1$; both have infinite support in $\mathrm{x}$ and $\mathrm{y}$, but are disjoint. The degree of the overlap between two distributions with infinite support can also be quantified using the Weitzman's measure.

\subsection{Comparison of assumptions using relational algebraic operators in CMdB}

If $P_{1}$ and $P_{2}$ are two polytopes in a relation $R$ in the $\mathrm{CMdB}$, then the relational algebraic operators are $P_{1} C P_{2}$ for subset, $P_{1} \partial P_{2}$ for disjoint and $P_{1} \cap P_{2}$ for intersection and they help facilitate decision analysis based on assumptions.

Let $P^{\prime}=\left\{P_{1}, P_{2}, \ldots . P_{n}\right\}$ be the set of all models/polytopes in a relation/table $P^{\prime}$ and $Q^{\prime}=\left\{Q_{1}, Q_{2}, \ldots . Q_{n}\right\}$ be the set of all models/polytopes in another relation/table $Q^{\prime}$. Then $P_{i}=\left\{c_{1}, c_{2}, \ldots c_{n}\right\}$ be the set of constraints/half spaces representing each polytope $P_{i}$. Also let $R_{i} \in R^{N}$ be the region enclosed by polytope $P_{i}$. Then $R_{1}=$ $\left\{x_{1}, x_{2}, \ldots . x_{n}\right\}$ where $x_{1}, x_{2} \ldots x_{n}$ are points lying in region $R_{1}$ of polytope $P_{1}$, only if countable. Similarly $R_{2}$ $=\left\{y_{1}, y_{2}, \ldots . y_{n}\right\}$ where $y_{1}, y_{2} \ldots y_{n}$ are points lying in region $R_{2}$ of polytope $P_{2}$.

1. A disjoint operation is defined by $P_{1} \partial P_{2}=\left\{x_{i} \in R_{1}\right.$ and $\left.y_{i} \in R_{2}=>x_{i} \neq y_{i}\right\}$

2. An intersect operation is defined by $P_{1} \cap P_{2}=\left\{\exists x_{i} \mid x_{i} \in R_{1}\right.$ and $\left.x_{i} \in R_{2}\right\}$

3. A subset operation is defined by $P_{1} C P_{2}=\left\{\forall x_{i} \mid x_{i} \in R_{1}\right.$ and $\left.x_{i} \in R_{2}\right\}$

We use algorithms briefly mentioned in section 2.1 based on Linear programming to compute these operations between polytopes and answer queries. Special query optimization techniques exist in the database to retrieve faster results since these queries involve heavy weight operations, namely solving LP's.

A sample query would be, SELECT * FROM T WHERE T. * INTERSECTS P

This query fetches from table $\mathrm{T}$, every polytope which intersects with polytope $\mathrm{P}$. This means that these polytope models have behavior similar to the model implied by $\mathrm{P}$, when the parameters are in the intersection region.

\section{APPLICATION FOR DECISION MAKING}

The CMdB finds use in a wide variety of applications ranging from supply chain management, energy optimization to transportation systems (Sanat 2018).

\subsection{Analysing Assumptions}

Consider an example from supply chain management where the CMdB is used for decision support. 
Example: Consider a distributor dealing with the distribution of 3 products, namely choco spread, jam and peanut butter in 3 different cities namely, C1, C2 and C3. The distributor has demand data for these products in all the three cities for the last 1 year. However, the demand in these cities or markets is uncertain and decision support is critically dependent on assumptions. The distributor would like to analyze his data and run different queries such as the ones listed below.

Q1: A new product which is a variant of peanut butter is being launched whose demand is based on assumptions. Is this demand similar to the demand of existing peanut butter product based on data?

Q2: For all the three products in the distributor's portfolios, are the demands in all three cities different?

Q3: Three different stakeholders provide different a-priori assumptions on the demands for city C1. Which assumptions should be considered while considering any future decision?

We have methods to derive models/constraint sets/polytopes from any historical data using an N-dimensional German tank method (Aswal et.al. 2011). Polytopes can also be created based on assumptions specified in the form of constraints. Using the $\mathrm{CMdB}$ we can systematically analyze relationships between summary polytopes derived from the demand data. For $Q 1$ above, four polytopes can be stored, three derived from demand data of peanut butter for each of the three cities and another based on assumptions for the demand of the new product. When checked for an intersecting relationship, if the polytope for the new product intersects with the demand data of any of the cities, then it indicates a good candidate city

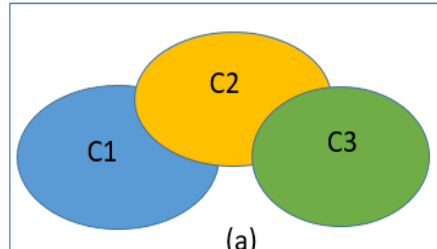

(a)

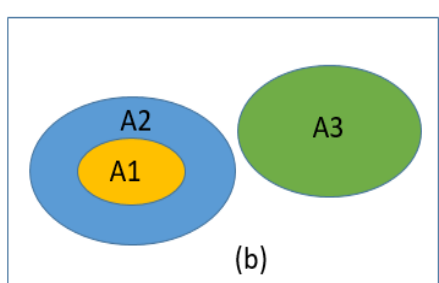

Figure 1. Relational algebraic analysis of convex uncertainty sets with assumptions to launch the new product.

Similarly, for $Q 2$, three polytopes can be derived representing all the data of the three products, one for each city. Let us say, when an algebraic relationship query is executed on them, their relationships are as shown in figure 1(a) where polytope $\mathrm{C} 1$ represents demand in city $C 1, \mathrm{C} 2$ for city $C 2$ and $\mathrm{C} 3$ for city $C 3$.This will provide insight to the distributor that the demand of the three products are similar between cities $C 1$ and $C 2$ and between $C 2$ and $C 3$ and that $C 1$ and $C 3$ demands are completely different from each other.

For query $Q 3$, each of the three polytopes based on assumptions of three different stakeholders are A1, A2 and A3 as shown in figure 1(b). Which input assumption set can we choose here? A3 being completely different, we may want to ignore it. In choosing polytope A1, we assume the least and in choosing polytope A2, we assume the most. Since A1 is a subset of A2, polytope A2 encompasses all the scenarios of A1 and some more. The database can also provide the information content in the polytopes in terms of number of bits calculated using Shannon's entropy (Shannon 1948), details of which are omitted for brevity. If the information content of A1 is close to that of A2, then A1 could be a good choice since it does not assume too many assumptions with similar information content. Thus without looking at the underlying microscopic data behind the different constraint sets or scenarios, we can analyze and attempt to identify the most suitable constraint set to drive our decisions quickly.

\subsection{Alternative scenario sets with varied assumptions}

Consider a small supply chain with two suppliers, two factories, two warehouses and two demand variables (real supply chains have many more dimensions). The demand variables cannot vary arbitrarily. Their variability is modelled as a region of the 2-dimensional demand space, defined by certain constraints. In this example, the constraints are linear, and forms a polytope, a special case of a convex model.

A hierarchy of constraint sets (forming a polytope) is generated from a given constraint set on the uncertain demand variables and we see how

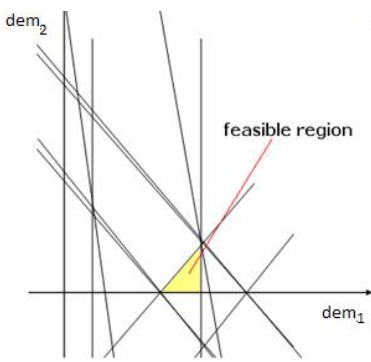

(a) Feasible region with first 10 constraints
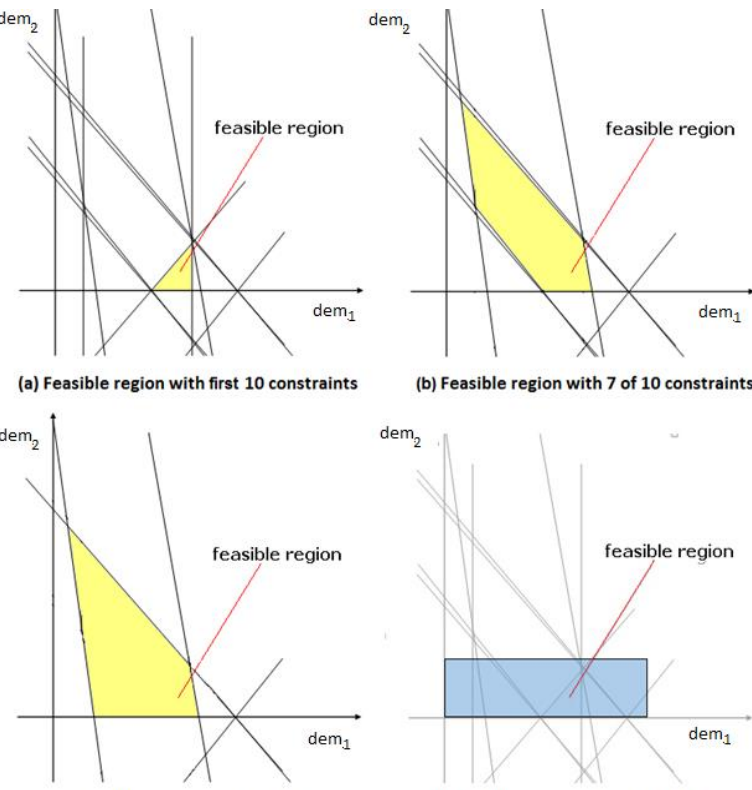

(b) Feasible region with 7 of 10 constraints

(c) Feasible region with 4 of 10 constraints

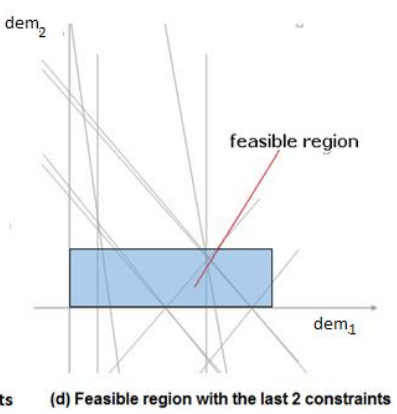

Figure 2. Hierarchy of constraints 
guarantees on the output become looser as uncertainty increases. We show below constraints derived for the 2 demand variables from demand data. dem $_{1}$ is the demand of product 1 in market 1 and dem $_{2}$ is the demand of product 1 in market 2 . These constraints are economically meaningful.

$$
\begin{gathered}
\text { c1: } 171.43 \text { dem }_{1}+128.57 \text { dem }_{2} \leq 79285.71 \\
\text { c2: } 171.43 \text { dem }_{1}+128.57 \text { dem }_{2} \geq 42857.14 \\
c 3: 57.14 \text { dem }_{1}+42.86 \text { dem }_{2} \leq 26428.57 \\
c 4: 57.14 \text { dem }_{1}+42.86 \text { dem }_{2} \geq 14285.71 \\
\text { c5: } 175.0 \text { dem }_{1}+25.0 \text { dem }_{2} \leq 65000.0 \\
\text { c6: } 175.0 \text { dem }_{1}+25.0 \text { dem }_{2} \geq 22500.0 \\
\text { c7: } 0.51 \text { dem }_{1}-0.39 \text { dem }_{2} \leq 237.86 \\
\text { c8: } 0.51 \text { dem }_{1}-0.39 \text { dem }_{2} \geq 128.57 \\
\text { c9: } 300.0 \text { dem }_{1} \leq 105000.0 \\
c 10: 300.0 \text { dem }_{1} \geq 30000.0
\end{gathered}
$$

Constraints 1 to 6 in order are bounds on the sum of product of demand and price. These are revenue constraints. Constraints 7 and 8 are competitive constraints and 9 and 10 are bounds on demand in market 1 . Figure 2 (a) shows the graphical representation of the constraint set formed with these constraints. Figure 2 (b) shows the graphical representation if constraints 8,9 and 10 are deleted from the set in Figure 2 (a). The volume of the feasible region increases signifying more uncertainty. These constraints represents the situation when only the revenue constraints are acting and the market is not competitive. Figure 2 (c) shows the constraint set if 3 more constraints are deleted. Figure 2 (d) shows the constraint set when only the bound constraints 9 and 10 are acting. This constraint set intersects with the constraint sets shown in Figures 2 (a), (b) and (c).

Table 1 summarizes the information content for the hierarchy of constraint sets formed by constraints 1 to 10 and also bounds for total cost (using optimization methods outside the scope of this paper), which is the objective function for this example. We have defined information content as the volume of the uncertain polyhedral set (Shannon 1948). As the uncertainty reduces, the bounds become tighter (range of output varies from $28 \%$ for 10 constraints to $106.85 \%$ for just two constraints).

Table 1. Summary of information analysis for hierarchical constraints

$\begin{array}{cccccc}\begin{array}{c}\text { Number of } \\ \text { constraints }\end{array} & \begin{array}{l}\text { Information Content } \\ \text { in No of bits }\end{array} & \begin{array}{l}\text { Minimum Cost } \\ (\%)\end{array} & \begin{array}{l}\text { Maximum } \\ (\%)\end{array} & \begin{array}{l}\text { Cost } \\ \text { Range } \\ \text { Uncertainty (\%) }\end{array} & \begin{array}{c}\text { of } \\ 10\end{array} \\ 1.84 & 100.00 & 128.38 & 28.38 \\ 9 & 0.81 & 60.06 & 154.50 & 94.45 \\ 7 & 0.73 & 60.06 & 158.72 & 98.66 \\ 4 & 0.58 & 54.99 & 158.72 & 103.73 \\ 2 & 0.44 & 54.92 & 161.77 & 106.85\end{array}$

This example illustrates how a hierarchy of scenario sets that hold economic meaning is generated, amount of uncertainty in the scenario sets is quantified, and how the change in the performance metric as the amount of uncertainty increases is examined. A decision support system supporting such an analysis has to offer facilities for specifying and optimizing over a hierarchy of constraint sets related through the subset and intersection operators. The CMdB proves useful for such analysis.

\subsection{Relational Algebraic Operator Results}

Table 2. Supply chain networks with time taken for relational algebraic operations between two polytopes

\begin{tabular}{c|ccccc}
\multicolumn{1}{c}{ Network Structure } & $\begin{array}{c}\text { Number of } \\
\text { Variables }\end{array}$ & $\begin{array}{c}\text { Number of } \\
\text { Constraints }\end{array}$ & $\begin{array}{c}\text { Disjoint Operation } \\
\text { Time }(\mathrm{ms})\end{array}$ & $\begin{array}{c}\text { Intersection } \\
\text { Time }(\mathrm{ms})\end{array}$ & $\begin{array}{c}\text { Subset } \\
\text { Time }(\mathrm{ms})\end{array}$ \\
\hline 3 Suppliers, 3 Factories, 3 & 20 & 50 & $<1$ & 15 & 25 \\
$\begin{array}{c}\text { Warehouses \& 3 Markets } \\
\text { 2 Suppliers, 2 Factories, 5 }\end{array}$ & 100 & 200 & 2 & 60 & 75 \\
Warehouses \& 20 Markets & & & & 140 & 150 \\
2 Suppliers, 5 Factories, & 100 & 400 & 2 & &
\end{tabular}


We present a summary of our results in Table 2 from executing the relational algebraic operators, namely, disjoint, intersection and subset operators in the $\mathrm{CMdB}$, for supply chain networks of different sizes. We assume that the network consists of only suppliers, factories, warehouses and markets to meet demands. The results here are presented with Gurobi 7 profiled on a machine having an Intel i5 processor at $2.50 \mathrm{GHz}$ and 16GB RAM. Our runtimes being less than a second, the results are encouraging.

\subsection{Other Applications - Transportation}

The CMdB has also been used in transportation networks such as railways where each polytope corresponds to data representing a single train. The variables are arrival and departure times at block sections (section between two stations) and constraints are bounds on their min and max values, correlations between times at adjacent block sections etc. Different scenarios can be generated by introducing assumptions such as delays into the arrival and departure times. A relational algebraic analysis can be performed on the polytopes to check if trains conflict along any route (Sanat 2018). If two polytopes intersect, then it is an indication that the corresponding trains are conflicting. This kind of analysis involving inter polytope relationships can detect flaws in existing schedules by detecting conflicting trains and have the potential to improve them.

\section{CONCLUSION AND FUTURE WORK}

Uncertainty can be of many kinds and the theory of probability may not be suitable for all the cases. In such cases, robustness of the answers is more important and $\mathrm{CMdB}$ is a step towards making decision analysis efficient in such cases. Usage of models, along with the support to query those using relational algebraic operators makes the $\mathrm{CMdB}$ a valuable addition into the field. In the current form, our work is limited to convex models of data. Though the discrete domain has been explored, more work needs to be done to extend the database to support the non-convex models that arise thereof. Efficient applications to probabilistic representations encounters computational difficulties and further work is required to develop fast approximations. While our work is not limited to single stage decision problems, we have not currently applied it to multi-stage decision problems and that is a direction for future work. Additionally, applications to large real world problems involving other domains is the next natural step to this work.

\section{REFERENCES}

Aswal, A., Perumal, G. and Prasanna, G.N.S (2011). "On estimating constraints for robust optimization of supply chains", INFORMS Annual Meeting.

Bankes, S. (1993). Exploratory modeling for policy analysis. Operations research, 41(3), 435-449.

Ben-Tal, A., \& Nemirovski, A. (2000). Robust solutions of linear programming problems contaminated with uncertain data. Mathematical programming, 88(3), 411-424.

Boyd, S., \& Vandenberghe, L. (2004). Convex optimization. Cambridge university press.

Bradley, R., \& Drechsler, M. (2014). Types of uncertainty. Erkenntnis, 79(6), 1225-1248.

Shannon, C. E. (1948). A mathematical theory of communication. Bell system technical journal, 27(3), 379423.

Lempert, R. J., Groves, D. G., Popper, S. W., \& Bankes, S. C. (2006). A general, analytic method for generating robust strategies and narrative scenarios. Management science, 52(4), 514-528.

Morgan, M. G. (2009). Best practice approaches for characterizing, communicating and incorporating scientific uncertainty in climate decision making. DIANE publishing.

Riesch, H. (2012). Levels of uncertainty. Handbook of risk theory: Epistemology, decision theory, ethics, and social implications of risk, 87-110.

Sanat, R., Dutt, T., Chandrababu, A., Abhilasha, A. and Prasanna, G.S. (2018). Optimizing Schedule of Trains in Context of a Large Railway Network. 21st International Conference on Intelligent Transportation Systems (ITSC) (pp. 1214-1220). IEEE.

Spiegelhalter, D. J., \& Riesch, H. (2011). Don't know, can't know: embracing deeper uncertainties when analysing risks. Philosophical Transactions of the Royal Society A: Mathematical, Physical and Engineering Sciences, 369(1956), 4730-4750.

Van der Heijden, K. (2011). Scenarios: the art of strategic conversation. John Wiley \& Sons.

Walker, W. E., Harremoës, P., Rotmans, J., Van Der Sluijs, J. P., Van Asselt, M. B., Janssen, P., \& Krayer von Krauss, M. P. (2003). Defining uncertainty: a conceptual basis for uncertainty management in model-based decision support. Integrated assessment, 4(1), 5-17. 